\title{
Calculating Effective Wilson Coefficients for Kaon Decays in Renormalization Scale $\mu=1 \mathrm{GeV}$
}

\author{
Khadijeh Ghasemi ${ }^{1}$, Somayeh Mehrjoo ${ }^{2}$ \\ ${ }^{1}$ Physics Department, Islamic Azad University, Central Tehran Branch, Tehran, Iran \\ ${ }^{2}$ Physics Department, Lorestan University, Lorestan, Iran
}

Email address:

kh.ghasemi86@gmail.com (Kh. Ghasemi),mehrjoo_ph84@yahoo.com (S. Mehrjoo)

\section{To cite this article:}

Khadijeh Ghasemi, Somayeh Mehrjoo. Calculating Effective Wilson Coefficients for Kaon Decays in Renormalization Scale $\mu=1 \mathrm{GeV}$. American Journal of Physics and Applications. Special Issue: Physics Quantum. Vol. 3, No. 6-1, 2015, pp. 1-5. doi: 10.11648/j.ajpa.s.2015030601.11

Received: September 25, 2015; Accepted: October 24, 2015; Published: March 14, 2016

\begin{abstract}
The decay rates of $K$ and $\bar{K}$ mesons, consisting of a quark-anti quark, as a weak decay in the presence of strong interactions have been studied by means of the Effective Hamiltonian Theory. One of the most important key factors for calculating Effective Hamiltonian is Wilson coefficients. In this paper, effective Wilson coefficients in renormalization scale $\mu=1 \mathrm{GeV}$ are calculated.
\end{abstract}

Keywords: K Meson, Effective Hamiltonian, Wilson Coefficients, CKM Matrix

\section{Introduction}

One of the successful models in particle phenomenology is the quark model which is applied to calculate the decays of various particles with a few differences. The particles called kaons, or K mesons, were first observed in the late 1940s in cosmic-ray experiments. By today's standards, they are common, easily produced, and well understood. Over the last four decades research into how kaons decay has played a major role in the development of the Standard Model. Yet, after all this time, kaon decays may still prove to be a valuable source of new information on some of the remaining fundamental questions in particle physics.

When first observed, kaons seemed quite mysterious. Experiments showed that they were produced in reactions involving the strong force, or strong interaction - the most powerful of the four fundamental forces in nature-but that they did not decay (that is, transform into two or more less massive particles) through the strong interaction. This is because kaons have a property, ultimately labeled "strangeness," which is conserved in the strong interaction [10].

One of the most interesting and unique observed particles in the nature is kaon. There are two neutral kaons which are, in fact, strange mesons.

$$
\begin{array}{ll}
\mathrm{k}^{\circ}=\mathrm{d} \overline{\mathrm{s}} & (\mathrm{s}=-1) \\
\overline{\mathrm{k}}^{\circ}=\mathrm{s} \overline{\mathrm{d}} & (\mathrm{s}=+1)
\end{array}
$$

$\mathrm{S}$ is the Eigenvalue of the strange state. Since each kaon under CP effect turns into another kaon, neither of these kaons have determined $\mathrm{CP}$ number. $\mathrm{k}^{\circ}$ and $\overline{\mathrm{k}}^{\circ}$ are not eigenstate of $\mathrm{CP}$. However, when $\mathrm{CP}$ acts on them, they are conjugate of each other.

$$
\begin{aligned}
& \mathrm{CP}\left|\mathrm{k}^{0}\right\rangle=-\left|\overline{\mathrm{k}}^{0}\right\rangle \\
& \mathrm{CP}\left|\overline{\mathrm{k}}^{\circ}\right\rangle=-\left|\mathrm{k}^{0}\right\rangle
\end{aligned}
$$

But theorists can make a pair kaon with determined $\mathrm{CP}$ from combination of wave function $\mathrm{k}^{\circ}$ and $\overline{\mathrm{k}}^{\circ}$.According to Quantum Mechanics rules, these combinations corresponding with real particles and have a mass and determined lifetime. Therefore normalized eigenstate $\mathrm{CP}$ are $[3,9]$ :

$$
\begin{aligned}
& \left|\mathrm{k}_{1}\right\rangle=\frac{1}{\sqrt{2}}\left(\left|\mathrm{k}^{\circ}\right\rangle-\left|\overline{\mathrm{k}}^{\circ}\right\rangle\right) \\
& \left|\mathrm{k}_{2}\right\rangle=\frac{1}{\sqrt{2}}\left(\left|\mathrm{k}^{\circ}\right\rangle+\left|\overline{\mathrm{k}}^{\circ}\right\rangle\right)
\end{aligned}
$$




$$
\begin{aligned}
& \mathrm{CP}\left|\mathrm{k}_{1}\right\rangle=\left|\mathrm{k}_{1}\right\rangle \quad(\mathrm{CP}=+1) \\
& \mathrm{CP}\left|\mathrm{k}_{2}\right\rangle=-\left|\mathrm{k}_{2}\right\rangle \quad(\mathrm{CP}=-1)
\end{aligned}
$$

$\mathrm{k}_{1}$ just can decays to $\mathrm{CP}=+1$ state, while $\mathrm{k}_{2}$ should go to $\mathrm{CP}=-1$ state. Neutral kaons usually decay to two or three pions. Arrangement of two pions has +1 parity and three pions system has -1 parity and both of them have a $\mathrm{C}=+1$. As a result, $\mathrm{k}_{1}$ decays to two pions and $\mathrm{k}_{2}$ decays to three pions [11].

$$
\begin{aligned}
& \mathrm{k}_{1} \rightarrow 2 \pi \\
& \mathrm{k}_{2} \rightarrow 3 \pi
\end{aligned}
$$

Since a kaon has hardly enough mass to produce three pions, two pion decays are fast but three pion decays are longer. Observed lifetimes are about $10^{-10} \mathrm{~s}$ and $10^{-7} \mathrm{~s}$, respectively $[3,12]$.

$\mathrm{K}$ mesons decay as a weak decay in the presence of strong interactions requires a special approach. The main tool to investigate these decays is the effective Hamiltonian theory. Beginning of any phenomenological weak decay of hadrons is the effective weak Hamiltonian that its structure is as follows $[4,6]$ :

$$
\mathrm{H}_{\text {eff }}=\frac{\mathrm{G}_{\mathrm{F}}}{\sqrt{2}} \sum_{\mathrm{i}} \mathrm{V}_{\mathrm{CKM}}^{\mathrm{i}} \mathrm{C}_{\mathrm{i}}(\mu) \mathrm{Q}_{\mathrm{i}}
$$

Where $G_{F}$ is the Fermi constant that in terms of the $g_{w}$ weak coupling constant and $\mathrm{W}$ boson mass is defined as follows:

$$
\frac{\mathrm{G}_{\mathrm{F}}}{\sqrt{2}}=\frac{\mathrm{g}_{\mathrm{w}}^{2}}{8 \mathrm{M}_{\mathrm{W}}^{2}}
$$

And $\mathrm{Q}_{\mathrm{i}}$ are the local operators that decays discussed in turn controlled. $\mathrm{V}_{\mathrm{CKM}}^{\mathrm{i}}$ Cabibbo - Kobayashi - Maskawa factors and $\mathrm{C}_{\mathrm{i}}$ Wilson Coefficients are described the force with which an operator enters the Hamiltonian. In fact, the effective point-like vertices are represented by local operators can correct picture of the decay of hadrons with a mass of the order of $\mathrm{O}\left(\mathrm{m}_{\mathrm{b}}, \mathrm{m}_{\mathrm{c}}\right)$ a better way to provide. $\mathrm{C}_{\mathrm{i}}$ The Wilson coefficients to be used as coupling constants (depending on scale) corresponding to the vertices are considered. Select the $\mu$ scale is optional, but it is customary that to choice $\mu$ the order of the mass of hadrons decay, eg for B and D mesons decays, the value of $\mu$ are respectively the order of $m_{b}$ and

$$
V_{\text {CKM }}=\left(\begin{array}{ccc}
c_{12} c_{13} & s_{12} c_{13} & s_{13} e^{-i \delta} \\
-s_{12} c_{23}-c_{12} s_{23} s_{13} e^{i \delta} & c_{12} c_{23}-s_{12} s_{23} s_{13} e^{i \delta} & s_{23} c_{13} \\
s_{12} s_{23}-c_{12} s_{23} s_{13} e^{i \delta} & -c_{12} s_{23}-s_{12} c_{23} s_{13} e^{i \delta} & c_{23} c_{13}
\end{array}\right)
$$

$\mathrm{m}_{\mathrm{c}}$. For kaon decays the common choice of $\mu$ is the order of $1-2 \mathrm{Gev}$ instead of $\mathrm{m}_{\mathrm{K}}$ order [1].

\section{Theoretical Framework}

In this paper, Wilson coefficients of $s$ quark and $\bar{s}$ antiquark decays are calculated [2]. General framework of how to calculate Wilson coefficients is based on that (1-6) equation which has already been mentioned in the introduction. Effective Hamiltonian of the $k \rightarrow \pi \pi$ transition is defined as follows [2].

$$
\mathrm{H}_{\text {eff }}(\Delta \mathrm{S}=1)=\frac{\mathrm{G}_{\mathrm{F}}}{\sqrt{2}} \mathrm{~V}_{\mathrm{ud}} \mathrm{V}_{\mathrm{us}}^{*}\left(\sum_{\mathrm{i}=1}^{10} \mathrm{C}_{\mathrm{i}}(\mu) \mathrm{Q}_{\mathrm{i}}(\mu)\right)+\text { h.c. }
$$

In this equation, $G_{F}$ is the Fermi constant and $Q_{i}$ is the local operator which controls the decay. $C_{i}$ coefficients are showed Wilson coefficients. The overall structure of the Wilson coefficients is as follow:

$$
\mathrm{C}_{\mathrm{i}}(\mu)=\mathrm{Z}_{\mathrm{i}}(\mu)+\tau \mathrm{y}_{\mathrm{i}}(\mu)
$$

In this equation $\tau$ is defined as follows:

$$
\tau=\frac{-\mathrm{V}_{\mathrm{td}} \mathrm{V}_{\mathrm{ts}}^{*}}{\mathrm{~V}_{\mathrm{ud}} \mathrm{V}_{\mathrm{us}}^{*}}
$$

In the $\tau$ equation $V_{t d}, V_{u s}, V_{t s}$ and $V_{u d}$ are the elements of the Cabibbo - Kobayashi - Maskawa matrix. Cabibbo Kobayashi - Maskawa matrix is a unitary matrix which contains information on the strength of flavor changing weak decays. Technically, it specifies the mismatch of quantum states of quarks when they propagate freely and when they take part in the weak interactions $[3,13]$.

$$
\mathrm{V}_{\mathrm{CKM}}=\left(\begin{array}{ccc}
\mathrm{V}_{\mathrm{ud}} & \mathrm{V}_{\mathrm{us}} & \mathrm{V}_{\mathrm{ub}} \\
\mathrm{V}_{\mathrm{cd}} & \mathrm{V}_{\mathrm{cs}} & \mathrm{V}_{\mathrm{cb}} \\
\mathrm{V}_{\mathrm{td}} & \mathrm{V}_{\mathrm{ts}} & \mathrm{V}_{\mathrm{tb}}
\end{array}\right)
$$

$C K M$ matrix is the $3 \times 3$ matrix, since there are three generations of quarks, which Kobayashi and Maskawa in 1973 stated that the third generation of quarks to the matrix, mixed phases that, if not zero, it is symmetry breaking. If this phase is virtually zero, to explain the CP failure must seek something beyond the standard model.

Several methods have been proposed for $C K M$ matrix parameterization which among them to discuss the introduction of standard parameterization.
In which, $\mathrm{c}_{\mathrm{ij}}=\cos \theta_{\mathrm{ij}}$ and $\mathrm{s}_{\mathrm{ij}}=\sin \theta_{\mathrm{ij}}$ for $\mathrm{i}, \mathrm{j}=1,2,3 . \delta$ is the phase which is in the range of $\circ \leq \delta \leq 2 \pi$. Matrix elements are calculated by using the following data [14]: 


$$
\begin{aligned}
& \theta_{12}=0.221 \\
& \theta_{13}=0.0035 \\
& \theta_{23}=0.041 \\
& \delta=\frac{\pi}{2} \\
& \mathrm{~V}_{\text {СКм }}=\left(\begin{array}{ccc}
0.9756 & 0.2192 & -0.0034 \mathrm{i} \\
-0.2190-0.000135 \mathrm{i} & 0.9747-0.000030 \mathrm{i} & 0.4089 \\
0.0089-0.003410 \mathrm{i} & -0.0399-0.000760 \mathrm{i} & 0.9990
\end{array}\right)
\end{aligned}
$$

To obtain $s$ quark decay rate, we need the effective Wilson coefficients of the tree and penguin decay. The effective Wilson coefficients can be defined as follows [3]:

$$
C_{i}^{\text {eff }}(\mu)=Z_{i}^{\text {eff }}(\mu)+\tau y_{i}^{\text {eff }}(\mu)
$$

Here $Z_{i}^{\text {eff }}(\mu)$ are defined as follows [2]:

$$
\begin{aligned}
& Z_{6}^{\text {eff }}(\mu)=Z_{6}(\mu)+\frac{\alpha_{s}}{4 \pi}\left(\gamma_{v}^{(0) \mathrm{T}} \ln \frac{\mu_{\mathrm{f}}}{\mu}+\hat{\mathrm{r}}_{\mathrm{v}}^{\mathrm{T}}\right)_{6 \mathrm{i}} \mathrm{Z}_{\mathrm{i}}(\mu)+\frac{\alpha_{\mathrm{s}}}{8 \pi}\left(\mathrm{C}_{\mathrm{t}}+\mathrm{C}_{\mathrm{p}}\right) \\
& Z_{7}^{\text {eff }}(\mu)=Z_{7}(\mu)+\frac{\alpha_{s}}{4 \pi}\left(\gamma_{v}^{(0) \mathrm{T}} \ln \frac{\mu_{\mathrm{f}}}{\mu}+\hat{\mathrm{r}}_{\mathrm{V}}^{\mathrm{T}}\right)_{7 \mathrm{i}} \mathrm{Z}_{\mathrm{i}}(\mu)+\frac{\alpha}{8 \pi} \mathrm{C}_{\mathrm{e}} \\
& Z_{8}^{\text {eff }}(\mu)=Z_{8}(\mu)+\frac{\alpha_{s}}{4 \pi}\left(\gamma_{\mathrm{v}}^{(0) \mathrm{T}} \ln \frac{\mu_{\mathrm{f}}}{\mu}+\hat{\mathrm{r}}_{\mathrm{V}}^{\mathrm{T}}\right)_{8 \mathrm{i}} Z_{\mathrm{i}}(\mu) \\
& Z_{9}^{\text {eff }}(\mu)=Z_{9}(\mu)+\frac{\alpha_{s}}{4 \pi}\left(\gamma_{v}^{(0) \mathrm{T}} \ln \frac{\mu_{\mathrm{f}}}{\mu}+\hat{\mathrm{r}}_{\mathrm{V}}^{\mathrm{T}}\right)_{9 \mathrm{i}} \mathrm{Z}_{\mathrm{i}}(\mu)+\frac{\alpha}{8 \pi} \mathrm{C}_{\mathrm{e}} \\
& Z_{10}^{\text {eff }}(\mu)=Z_{10}(\mu)+\frac{\alpha_{s}}{4 \pi}\left(\gamma_{\mathrm{v}}^{(0) \mathrm{T}} \ln \frac{\mu_{\mathrm{f}}}{\mu}+\hat{\mathrm{r}}_{\mathrm{v}}^{\mathrm{T}}\right)_{10 \mathrm{i}} Z_{\mathrm{i}}(\mu) \\
& Z_{1}^{\text {eff }}(\mu)=Z_{1}(\mu)+\frac{\alpha_{s}}{4 \pi}\left(\gamma_{v}^{(0) \mathrm{T}} \ln \frac{\mu_{\mathrm{f}}}{\mu}+\hat{\mathrm{r}}_{\mathrm{V}}^{\mathrm{T}}\right)_{1 \mathrm{i}} Z_{\mathrm{i}}(\mu) \\
& Z_{2}^{\text {eff }}(\mu)=Z_{2}(\mu)+\frac{\alpha_{s}}{4 \pi}\left(\gamma_{v}^{(0) \mathrm{T}} \ln \frac{\mu_{\mathrm{f}}}{\mu}+\hat{\mathrm{r}}_{\mathrm{V}}^{\mathrm{T}}\right)_{2 \mathrm{i}} Z_{\mathrm{i}}(\mu) \\
& Z_{3}^{\text {eff }}(\mu)=Z_{3}(\mu)+\frac{\alpha_{s}}{4 \pi}\left(\gamma_{v}^{(0) \mathrm{T}} \ln \frac{\mu_{\mathrm{f}}}{\mu}+\hat{\mathrm{r}}_{\mathrm{v}}^{\mathrm{T}}\right)_{3 \mathrm{i}} \mathrm{Z}_{\mathrm{i}}(\mu)-\frac{\alpha_{\mathrm{s}}}{24 \pi}\left(\mathrm{C}_{\mathrm{t}}+\mathrm{C}_{\mathrm{p}}\right) \\
& Z_{4}^{\text {eff }}(\mu)=Z_{4}(\mu)+\frac{\alpha_{s}}{4 \pi}\left(\gamma_{V}^{(0) \mathrm{T}} \ln \frac{\mu_{f}}{\mu}+\hat{r}_{V}^{T}\right)_{4 i} Z_{i}(\mu)+\frac{\alpha_{s}}{8 \pi}\left(C_{t}+C_{p}\right) \\
& Z_{5}^{\text {eff }}(\mu)=Z_{5}(\mu)+\frac{\alpha_{s}}{4 \pi}\left(\gamma_{v}^{(0) \mathrm{T}} \ln \frac{\mu_{\mathrm{f}}}{\mu}+\hat{\mathrm{r}}_{\mathrm{V}}^{\mathrm{T}}\right)_{5 \mathrm{i}} \mathrm{Z}_{\mathrm{i}}(\mu)-\frac{\alpha_{\mathrm{s}}}{24 \pi}\left(\mathrm{C}_{\mathrm{t}}+\mathrm{C}_{\mathrm{p}}\right)
\end{aligned}
$$

$$
\gamma^{(0)}=\left(\begin{array}{cccccccccc}
-2 & 6 & 0 & 0 & 0 & 0 & 0 & 0 & 0 & 0 \\
6 & -2 & 0 & 0 & 0 & 0 & 0 & 0 & 0 & 0 \\
0 & 0 & -2 & 6 & 0 & 0 & 0 & 0 & 0 & 0 \\
0 & 0 & 6 & -2 & 0 & 0 & 0 & 0 & 0 & 0 \\
0 & 0 & 0 & 0 & 2 & -6 & 0 & 0 & 0 & 0 \\
0 & 0 & 0 & 0 & 0 & -16 & 0 & 0 & 0 & 0 \\
0 & 0 & 0 & 0 & 0 & 0 & 2 & -6 & 0 & 0 \\
0 & 0 & 0 & 0 & 0 & 0 & 0 & -16 & 0 & 0 \\
0 & 0 & 0 & 0 & 0 & 0 & 0 & 0 & -2 & 6 \\
0 & 0 & 0 & 0 & 0 & 0 & 0 & 0 & 0 & -2
\end{array}\right)
$$

In these equations, $T$ is matrix transpose operator. Matrix with variable dimensions $\gamma_{V}^{(0)}$ and constant matrix $\hat{\mathrm{r}}_{\mathrm{v}}$ are obtained from correction of $Q_{1}-Q_{10}$ operators' vertex. Also, the values of $C_{e}, C_{p}$ and $C_{t}$ are extracted from $Q C D$ Penguin diagram, $Q_{1,2}$ operators and QCD Penguin diagram, $Q_{3}-$ $Q_{6}$ operators and electroweak penguin diagrams $Q_{7}-Q_{10}$, respectively.

$$
\begin{aligned}
& \mathrm{C}_{\mathrm{t}}=\tilde{\mathrm{G}}\left(\mathrm{m}_{\mathrm{u}}\right) \mathrm{Z}_{1} \\
& \mathrm{C}_{\mathrm{p}}=\left[\tilde{\mathrm{G}}\left(\mathrm{m}_{\mathrm{s}}\right)+\tilde{\mathrm{G}}\left(\mathrm{m}_{\mathrm{d}}\right)\right] \mathrm{Z}_{3}+\sum_{\mathrm{i}=u, d, \mathrm{~s}} \tilde{\mathrm{G}}\left(\mathrm{m}_{\mathrm{i}}\right)\left(\mathrm{Z}_{4}+\mathrm{Z}_{6}\right) \\
& \mathrm{C}_{\mathrm{e}}=\frac{8}{9} \tilde{\mathrm{G}}\left(\mathrm{m}_{\mathrm{u}}\right)\left(\mathrm{Z}_{1}+3 \mathrm{Z}_{2}\right) \\
& \tilde{\mathrm{G}}\left(\mathrm{m}_{\mathrm{q}}\right)=\frac{2}{3} \kappa-\mathrm{G}\left(\mathrm{m}_{\mathrm{q}}, \mathrm{k}, \mu\right)
\end{aligned}
$$

In which, $\kappa$ is parameter that in dimensional regulation, describes dependency $\gamma_{5}$ model. For example, in $\gamma_{5}$ model we have Naïve Dimensional Regularization (NDR) and Hooft-Veltman $(H V)$ :

$$
\kappa= \begin{cases}1 & \mathrm{NDR} \\ 0 & \mathrm{HV}\end{cases}
$$

Function $G(m, k, \mu)$ in (2-6) equations is defined as follows:

$$
G(m, k, \mu)=-4 \int_{0}^{1} d x x(1-x) \ln \left(\frac{m^{2}-k^{2} x(1-x)}{\mu^{2}}\right)
$$

In which $k^{2}$ is the square of the momentum carried by virtual gluons. $\hat{r}$ Matrix in (2-9) equations gives constant terms; they are independent of the momentum that is based on the $\gamma_{5}$ behavior in the dimensional regularization. For the kaon decays, there was no heavy quark mass scale between $m_{c}$ and $m_{K}$. Therefore, logarithmic term arising from corrections four quarks operations vertex to $\ln \mu_{f} / \mu$ form will be like (2-9) equation. We will assume that $\mu_{f}=1 \mathrm{GeV}$ as a reliable estimate obtained of destruction effects the effective Wilson coefficients. As a result, we have [2, 4]: 


$$
\begin{aligned}
& \hat{\mathrm{r}}_{\mathrm{NDR}}=\left(\begin{array}{cccccccccc}
3 & -9 & 0 & 0 & 0 & 0 & 0 & 0 & 0 & 0 \\
-9 & 3 & 0 & 0 & 0 & 0 & 0 & 0 & 0 & 0 \\
0 & 0 & 3 & -9 & 0 & 0 & 0 & 0 & 0 & 0 \\
0 & 0 & -9 & 3 & 0 & 0 & 0 & 0 & 0 & 0 \\
0 & 0 & 0 & 0 & -1 & 3 & 0 & 0 & 0 & 0 \\
0 & 0 & 0 & 0 & -3 & 1 & 0 & 0 & 0 & 0 \\
0 & 0 & 0 & 0 & 0 & 0 & -1 & 3 & 0 & 0 \\
0 & 0 & 0 & 0 & 0 & 0 & -3 & 1 & 0 & 0 \\
0 & 0 & 0 & 0 & 0 & 0 & 0 & 0 & 3 & -9 \\
0 & 0 & 0 & 0 & 0 & 0 & 0 & 0 & -9 & 3
\end{array}\right) \\
& \hat{\mathrm{r}}_{\mathrm{HV}}=\left(\begin{array}{cccccccccc}
7 / 3 & -7 & 0 & 0 & 0 & 0 & 0 & 0 & 0 & 0 \\
-7 & 7 / 3 & 0 & 0 & 0 & 0 & 0 & 0 & 0 & 0 \\
0 & 0 & 7 / 3 & -7 & 0 & 0 & 0 & 0 & 0 & 0 \\
0 & 0 & -7 & 7 / 3 & 0 & 0 & 0 & 0 & 0 & 0 \\
0 & 0 & 0 & 0 & -3 & 9 & 0 & 0 & 0 & 0 \\
0 & 0 & 0 & 0 & 1 & -1 / 3 & 0 & 0 & 0 & 0 \\
0 & 0 & 0 & 0 & 0 & 0 & -3 & 9 & 0 & 0 \\
0 & 0 & 0 & 0 & 0 & 0 & 1 & -1 / 3 & 0 & 0 \\
0 & 0 & 0 & 0 & 0 & 0 & 0 & 0 & 7 / 3 & -7 \\
0 & 0 & 0 & 0 & 0 & 0 & 0 & 0 & -7 & 7 / 3
\end{array}\right)
\end{aligned}
$$

\section{Conclusion}

Table 1. $Z_{i}^{\text {eff }}$ in renormalization scale $\mu=1 \mathrm{GeV}$.

\begin{tabular}{lllll}
\hline & NDR & HV & $\mathbf{Z}_{\mathbf{i}}^{\text {eff }}(\mathbf{N D R})$ & $\mathbf{Z}_{\mathbf{i}}^{\text {eff }}(\mathbf{H V})$ \\
\hline $\mathrm{Z}_{1}$ & 1.278 & 1.371 & 1.718 & 1.713 \\
$\mathrm{Z}_{2}$ & -0.509 & -0.640 & -1.113 & -1.110 \\
$\mathrm{Z}_{3}$ & 0.013 & 0.007 & 0.032 & 0.032 \\
$\mathrm{Z}_{4}$ & -0.035 & -0.017 & -0.081 & -0.084 \\
$\mathrm{Z}_{5}$ & 0.008 & 0.004 & 0.024 & 0.025 \\
$\mathrm{Z}_{6}$ & -0.035 & -0.014 & -0.086 & -0.086 \\
\hline
\end{tabular}

Table 2. $y_{i}^{\text {eff }}$ in renormalization scale $\mu=1 \mathrm{GeV}$.

\begin{tabular}{lllll}
\hline & NDR & HV & $\mathbf{y}_{\mathbf{i}}^{\text {eff }}(\mathbf{N D R})$ & $\mathbf{y}_{\mathbf{i}}^{\text {eff }}(\mathbf{H V})$ \\
\hline $\mathrm{y}_{1}$ & 0 & 0 & 0 & 0 \\
$\mathrm{y}_{2}$ & 0 & 0 & 0 & 0 \\
$\mathrm{y}_{3}$ & 0.031 & 0.036 & 0.050 & 0.049 \\
$\mathrm{y}_{4}$ & -0.056 & -0.059 & -0.053 & -0.053 \\
$\mathrm{y}_{5}$ & -0.001 & 0.016 & 0.003 & 0.002 \\
$\mathrm{y}_{6}$ & -0.109 & -0.096 & -0.160 & -0.138 \\
\hline
\end{tabular}

Table 3. The effective Wilson coefficients in renormalization scale $\mu=1 \mathrm{GeV}$.

\begin{tabular}{lll}
\hline & $\mathbf{C}_{\mathbf{i}}^{\text {eff }}(\mathbf{N D R})$ & $\mathbf{C}_{\mathbf{i}}^{\text {eff }}(\mathbf{H V})$ \\
\hline $\mathrm{C}_{1}^{\text {eff }}$ & 1.718 & 1.713 \\
$\mathrm{C}_{2}^{\text {eff }}$ & -1.113 & -1.110 \\
$\mathrm{C}_{3}^{\text {eff }}$ & $0.0320834-0.0000335139 \mathrm{i}$ & $0.0320817-0.0000328436 \mathrm{i}$ \\
$\mathrm{C}_{4}^{\text {eff }}$ & $-0.0810884+0.0000355247 \mathrm{i}$ & $-0.0840884+0.0000355247 \mathrm{i}$ \\
$\mathrm{C}_{5}^{\text {eff }}$ & $0.024005-2.01083 \times 10^{-6} \mathrm{i}$ & $0.0250033-1.34056 \times 10^{-6} \mathrm{i}$ \\
$\mathrm{C}_{6}^{\text {eff }}$ & $-0.0862669+0.000107244 \mathrm{i}$ & $-0.0862302+0.0000924984 \mathrm{i}$ \\
\hline
\end{tabular}

By using the effective Lagrangian density of the weak interaction, we can calculate decay rate in tree level. Furthermore, decay rates of $\mathrm{S}$ quark -anti quark can be calculated in tree and penguin level by the use of the effective Hamiltonian Theory. This is possible by the means of Effective Wilson coefficients.

In this paper, Effective Wilson coefficients are calculated. In table $1 Z_{i}^{\text {eff }}$ values were calculated. Moreover, the numerical values of $y_{i}^{\text {eff }}$ are showed in the table 2 [5]. In conclusion, Table 3 shows calculated values for the effective Wilson coefficients for the decay of $s$ quark and $\bar{s}$ antiquark in renormalization scale $\mu=1 \mathrm{GeV}$. 


\section{References}

[1] Andrzej J. B., "Operator Product Expansion, Renormalization Group and Week Decays”, Lect. Notes. Phys. 558, pp. 65-85 (2000).

[2] Cheng, H. Y., “The Rule In Kaon Decays", Chinese Journal Of Physics, 38, 4 (2000).

[3] M. Ghabakhlou, "CP Violation in b quark decays", "M. Sc" Thesis, Semnan University, 1387.

[4] Buchalla, G., Andrzej J. B., Lautenbacher, M. E., "Weak Decays Beyond Leading Logarithms", Rev. Mod. Phys., 86, 4 (Oct 1996).

[5] Cheng, H. Y., "Factorization Approach For The Rule and In Kaon Decays", Chin. J. Phys., 38, (2000).

[6] H. Mehraban, M. Sayahi, "QCD FACTORIZATION IN HADRONIC $B \rightarrow J / \psi(\pi, K)$ DECAYS", Iranian Journal of Science \& Technology, Transaction A, Vol. 34, No. A4 (2010).

[8] H. Mehraban, "Effective Hamiltonian and Effective Penguin Model on b Quark Decays", Transaction B: Mechanical Engineering, Vol. 16, No. 5, pp. $371\{386\}$, (October 2009).

[9] K. K. Vos, J. P. Noordmans, H. W. Wilschut and R. G. E. Timmermans1, "Exploration of Lorentz violation in neutralkaon decay", arxiv.org/abs/1401.2245v1,( 10 Jan 2014).

[10] J. Ritche "Rare kaon decays" BEAM LINE, V.2 5, N. 4, Winter 1995.

[11] Griffihs, D. J., "Introduction to Elementary Particles", John Wiley \& Sons (1987).

[12] Halkiadakis, E., "Measurements of Neutral Kaon Decays To Two Electron-Positron", University of New Brunswick, New Jersey (May 2001).

[13] Halzen, F., Martin, A. D., "Quarks and Leptons: An Introductory Course in Modern Particle Physics", John Wiley \& Sons (1984).

[14] Amster, C., "Particle Data Group", PLB667 (2008), J. Beringer et al. (Particle Data Group), PR D86, 010001 (2012).

[7] V. Cirigliano, G. Ecker, H. Neufeld, A. Pich and J. Portol'es, "Kaon Decays in the Standard Model", arXiv: 1107.6001v3, (14 Apr 2012). 